\title{
GMR
}

\section{Preparation of polyclonal antibody against porcine beta defensin 2 and identification of its distribution in tissues of pig}

\author{
Y.Y. Bao*, L. Li*, H. Zhang, C.Y. Gao, C.B. Xiao and C.L. Li \\ Department of Animal and Veterinary Science, Henan Agricultural University, \\ Zhengzhou, Henan, China \\ *These authors contributed equally to this study. \\ Corresponding author: C.L. Li \\ E-mail: hncll@163.com
}

Genet. Mol. Res. 14 (4): 18863-18871 (2015)

Received August 10, 2015

Accepted October 29, 2015

Published December 28, 2015

DOI http://dx.doi.org/10.4238/2015.December.28.36

\begin{abstract}
Porcine $\beta$-defensin 2 (pBD2) is an antimicrobial peptide in pigs that plays an important role in the immune system by preventing bacterial invasion. To produce an anti-pBD2 antibody, which is not commercially available, we expressed and purified a soluble, his-tagged version of pBD2 (his-pBD2). Purified pBD2 was injected into New Zealand white rabbits to generate polyclonal antiserum. Anti-pBD2 antibodies were purified by ammonium sulfate precipitation, followed by diethylaminoethyl cellulose ionexchange chromatography. The purified polyclonal antibody showed high sensitivity, with a titer as high as 204,800 by enzyme-linked immunosorbent assay, and it also showed high specificity for both his-pBD2 and native pBD2, as assessed by western blotting. Furthermore, immunohistochemistry analysis using the purified antibody revealed that pBD2 protein is distributed in the tongue, liver, kidney, small intestine, and large intestine of pigs. These results indicate that the prepared polyclonal antibody will be a useful tool for further studies of the function and mechanism of pBD2.
\end{abstract}

Key words: Porcine $\beta$-defensin 2; Polyclonal antibody; ELISA; Western blotting; Immunohistochemistry 


\section{INTRODUCTION}

Defensins are a large family of cationic antimicrobial peptides that comprise of 30-50 amino acid residues and exist widely in various species of plants and animals (Hancock, 2001; Ganz, 2003; Ganz, 2004; Selsted and Ouellette, 2005; Klüver et al., 2006). They have broad antimicrobial activity against pathogens, including bacteria, viruses, and fungi (Selsted and Ouellette, 2005; Peschel and Sahl, 2006; Yang et al., 2007; Auvynet and Rosenstein, 2009). In addition, they act as signaling molecules in the immune system, function as chemoattractants for memory T cells, immature dendritic cells, and mast cells, and are inflammatory mediators (Yang et al., 1999; Nozdrachev et al., 2006; Choi et al., 2012).

Mammalian defensins are classified as $\alpha, \beta$, and $\theta$ defensins based on the connectivity of three disulfide bridges (Lehrer and Ganz, 2002; Selsted and Ouellette, 2005). Evolutionarily, b-defensins are the oldest of the defensins (Sang et al. 2006). In pigs, only $\beta$-defensins have been found, which were all discovered by genomic sequencing analysis, except for porcine $\beta$-defensin 1 (pBD1). Few studies have been conducted on porcine defensins (Shi et al., 1999; Zhang et al., 2000; Sang et al., 2009). Previous research showed that porcine $\beta$-defensin 2 (pBD2) has high antimicrobial activity against Gram-negative and Gram-positive bacteria, including multidrug-resistant bacteria isolated from sick fowl, which makes it a good candidate for antibiotic development (Zhang et al., 2000; Veldhuizen et al., 2008; Li et al., 2013a). However, the antibacterial mechanism of pBD2 and its role in the immune system, remain unclear. Currently, no antipBD2 polyclonal antibody is commercial available for research. Thus, in this study, we prepared and characterized an anti-pBD2 polyclonal antibody. To generate an anti-pBD2 polyclonal antibody, we used a previously described strain, BL21(DE3)-pET-pBD2 (Gao et al., 2015), to express a histagged pBD2 (his-pBD2) fusion protein. Polyclonal antibody was prepared by injecting purified hispBD2 into New Zealand white rabbits. The antibody was then characterized using ELISAs, western blots, and immunohistochemistry experiments.

\section{MATERIAL AND METHODS}

\section{Expression and purification of his-pBD2}

His-pBD2 was expressed by inducing the Escherichia coli strain BL21(DE3)-pET-pBD2, which was constructed in our laboratory, with isopropyl $\beta$-D-1-thiogalactopyranoside (IPTG), and the protein was purified as previously described (Li et al., 2013a; Gao et al., 2015). The purified his-pBD2 was analyzed by SDS-PAGE, and used for the preparation of antibodies.

\section{Preparation and purification of polyclonal antibody against pBD2}

Polyclonal antibody was prepared followed previously reported protocols, with some modification (Liu et al., 2009; Liu et al., 2013; Li et al., 2013b). In brief, purified his-pBD2 protein was mixed with an equal volume of Freund's complete adjuvant (Sigma-Aldrich, St. Louis, MO, USA) at a final concentration of $300 \mu \mathrm{g} / \mathrm{mL}$. Subsequently, about $100 \mu \mathrm{g}$ his-pBD2 was injected subcutaneously into the backs of New Zealand white rabbits (3-months old, about $1.5-2 \mathrm{~kg}$ ). After two weeks, the rabbits were immunized again with $100 \mu \mathrm{g}$ his-pBD2 in Freund's incomplete adjuvant (Sigma-Aldrich). The third and fourth immunizations were executed with his-pBD2 in Freund's incomplete adjuvant at 2-week intervals. Blood was collected 7 days after the last injection. 
The blood was placed at room temperature for $1 \mathrm{~h}$, and then at $4^{\circ} \mathrm{C}$ overnight, and the supernatants (antisera) were collected via centrifugation at 2,200 $\mathrm{g}$ for $10 \mathrm{~min}$. Then, the antisera were mixed with an equal volume of saline solution, and precipitated with $20 \%$ saturated ammonium sulfate. After centrifugation at 3,500 rpm for $30 \mathrm{~min}$, the supernatants were collected and precipitated with $50 \%$ saturated ammonium sulfate. The final precipitates were dissolved in saline solution, precipitated with $33 \%$ saturated ammonium sulfate, and dissolved in saline solution again. The last two steps were repeated three times. Finally the primary, purified antibodies were desalinated by dialysis against PB buffer $(10 \mathrm{mM}, \mathrm{pH} 7.2)$ until no $\mathrm{NH}_{4}^{+}$could be detected. Then, the IgG fraction was further purified by diethylaminoethyl (DEAE)-cellulose chromatography (Parham et al., 1982); bound antibodies were eluted using $10 \mathrm{mM}$ PB buffer (10 mM, pH 7.2). The eluted fractions were collected and their absorbance at $280 \mathrm{~nm}$ was analyzed. The fractions with the highest absorbance values were pooled, concentrated using polyethylene glycol (PEG) 8000 , and identified by SDS-PAGE.

\section{Titer determination by ELISA}

The titer of purified anti-pBD2 antibody was determined by indirect ELISA according to previously described protocols, with some modifications (Kroll et al., 2005; Wu et al., 2007; Gao et al., 2012; Xiong et al. 2012; Xin et al., 2013). First, 96-well plates were coated with $100 \mu \mathrm{L}$ of the purified hispBD2 antigen, diluted to $20 \mathrm{ng} / \mathrm{mL}$ in $0.05 \mathrm{M}$ sodium carbonate coating buffer ( $\mathrm{pH} 9.6)$, at $4^{\circ} \mathrm{C}$ overnight, and washed three times with phosphate-buffered saline (PBS)-T buffer (0.05\% Tween 20 in PBS, pH 7.4). The plates were then blocked with $300 \mu \mathrm{L} 1 \%$ bovine serum albumen (BSA) for $1 \mathrm{~h} 37^{\circ} \mathrm{C}$. After washing three times with PBS-T, the purified anti-pBD2 antibody was used as a primary antibody at different dilutions (from $1: 400$ to $1: 819,200$ ) and incubated at $37^{\circ} \mathrm{C}$ for $1 \mathrm{~h}$; negative serum at the same dilution was used as a control. Plates were then incubated at $37^{\circ} \mathrm{C}$ for $1 \mathrm{~h}$ with $100 \mu \mathrm{L}$ of horseradish peroxidase (HRP)-labeled goat anti-rabbit IgG (1:5,000, DingGuo ChangSheng Biotechnology Co., Ltd., Beijing, China) as a secondary antibody. Peroxidase activity was detected at $37^{\circ} \mathrm{C}$ for 15 min using fresh tetramethylbenzidine (TMB) as a substrate, and the color development was stopped via the addition of $50 \mu \mathrm{L} 2 \mathrm{M} \mathrm{H}_{2} \mathrm{SO}_{4}$. The absorbance at $450 \mathrm{~nm}$ was measured using a microplate reader (Stat Fax 2100, Awareness Technologies Inc., Westport, CT, USA). The experiments were repeated three times, and all values are presented as the mean \pm standard deviation. The antibody titer was defined as the highest dilution of serum at which the absorbance ratio at $450 \mathrm{~nm}$ of the purified antibody and pre-immunization serum was greater than 2:1.

\section{Western blot analysis}

To confirm that the antibody could recognize the pBD2 protein in bacteria and in pigs, western blots were performed. Total proteins from the induced BL21(DE3)-pET-pBD2 strain and from strain BL21(DE3) were used as positive and negative controls, respectively. Total protein samples from porcine kidney tissues were used. Western blotting was performed according to a previously reported protocol (Xiong et al., 2012). Different fractions from different samples were separated by $10 \%$ SDS-PAGE, and transferred to a nitrocellulose membrane. The membrane was blocked for $2 \mathrm{~h}$ with $5 \%$ skimmed milk (Inner Mongolia Yili Industrial Group Co., Ltd., Inner Mongolia, China) in TBS-T buffer (0.05\% Tween-20 in TBS, pH 7.5) at room temperature. After three washes with TBST buffer (5 min each), the nitrocellulose membrane was incubated with the purified antipBD2 polyclonal antibody (1:3,000 dilution) at $4^{\circ} \mathrm{C}$ overnight. Then, the membrane was incubated 
with HRP-labeled goat anti-rabbit IgG at a dilution of 1:5000 for $2 \mathrm{~h}$ at room temperature. Finally, bound secondary antibody was detected using the Super ECL Plus Detection Reagent (Zhongshan biotechnology Co., Ltd., Beijing, China).

\section{Immunohistochemistry analysis}

Immunohistochemistry was performed according to a previously reported protocol, with some modifications (Liu et al., 2009). Tongue, liver, kidney, small intestine (including duodenum and jejunum), and large intestine (rectum) tissues from Large White piglets (about 10 days old) were selected and cut into approximately $1-\mathrm{cm}^{3}$ pieces. The samples were suspended and fixed in Bouin's solution for $24 \mathrm{~h}$ at room temperature, and then washed and dehydrated in a series of ethanol gradients. Subsequently, samples were embedded in paraffin wax and cut into approximately $5-\mu \mathrm{m}^{2}$ sections. The sections were hydrated by a series of ethanol-to-water gradients and treated with $3 \%$ hydrogen peroxide for $10 \mathrm{~min}$, incubated in citrate buffer for $10 \mathrm{~min}$, and incubated with 50 $\mu \mathrm{L}$ anti-goat serum for $30 \mathrm{~min}$ at room temperature. Subsequently, the sections were incubated with the purified anti-pBD2 polyclonal antibody at $4^{\circ} \mathrm{C}$ overnight (1:1000). After washing three times with PBS-T, tissue sections were incubated with HRP-labeled goat anti-rabbit IgG for 60 min at $37^{\circ} \mathrm{C}$ (1:500) and washed with PBS-T. Finally, the sections were stained with diaminobenzidine (DAB) and counterstained with hematoxylin, dehydrated, and mounted. Brown staining indicates the presence of pBD2. Serum from non-immunized rabbits served as a negative control for the primary antibody.

\section{RESULTS}

\section{Purification of his-pBD2}

Based on BL21(DE3)-pET-pBD2, which was constructed in our lab, his-pBD2 was expressed and purified as previously described (Li et al., 2013a; Gao et al., 2015). The molecular weight of purified his-pBD2 was about $12 \mathrm{kDa}$, and the purity was greater than $90 \%$ according to Gel-ProAnalyzer (4.0) analysis (Figure 1). The results showed that purified his-pBD2 could be used as antigen to immunize New Zealand white rabbits.

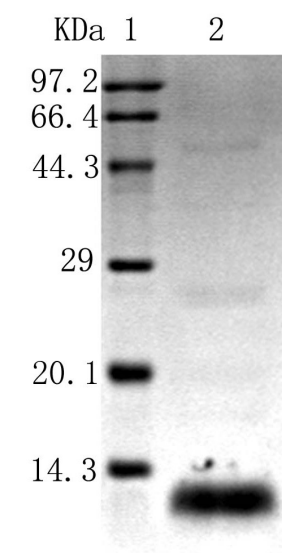

Figure 1. SDS-PAGE analysis of purified, his-tagged pBD2. Lane 1 shows the protein marker and lane 2 shows purified pBD2. 


\section{Purification of polyclonal antibody against pBD2}

After the rabbits were immunized four times with purified his-pBD2, polyclonal antibody against pBD2 was first precipitated with different concentrations of ammonium sulfate, and further purified by DEAE-cellulose ion-exchange chromatography. The purified antibody showed high purity and consisted of two bands, one was the heavy chain (about $52.5 \mathrm{kDa}$ ) and the other was the light chain (about $22.8 \mathrm{kDa}$ ), as assessed by SDS-PAGE and analyzed by Gel-Pro Analyzer (4.0), which were close in size to their predicted molecular weights (Figure 2).

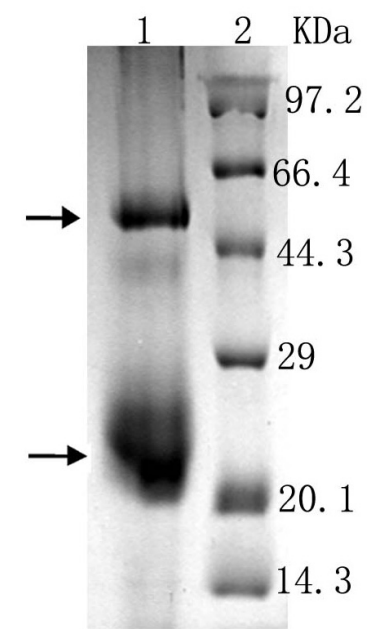

Figure 2. SDS-PAGE analysis of purified polyclonal antibody. Lane 1 shows the purified anti-pBD2 antibody and lane 2 shows the protein marker. Arrows indicate the heavy and light chains of the antibody.

\section{Titer analysis by ELISA}

The titer of the purified anti-pBD2 antibody was measured by ELISA. The polyclonal antibody was diluted by double dilution methods (from 400- to 819,200-fold) and reacted with 100 $\mu \mathrm{L} 20 \mathrm{ng} / \mathrm{mL}$ his-pBD2. Pre-immunized rabbit serum was used as a negative control. The titer of purified antibody was 204,800 , which indicated that the purified polyclonal antibody had good sensitivity for pBD2 (Figure 3).

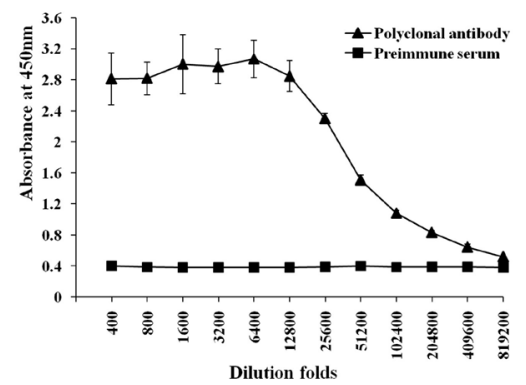

Figure 3. Titer of the purified anti-pBD2 antibody as determined by ELISA. Absorbance values at $450 \mathrm{~nm}$ of different dilutions of purified anti-pBD2 antibody and pre-immune serum respectively, are shown. 


\section{Western blot analysis}

The specificity of the purified antibody was analyzed by western blotting. Different samples were separated by $10 \%$ SDS-PAGE and transferred to nitrocellulose membranes (Figure 4). Predicted bands representing $\mathrm{pBD} 2$ were found in total protein samples from BL21(DE3)-pET-pBD2 and kidney tissue from pig, while proteins from the BL21(DE3) E. coli strain did not generate any bands. These results indicate that the polyclonal antibody did not react with any $E$. coli proteins; however, it could recognize $\mathrm{pBD} 2$, whether it was the his-tagged fusion protein in $E$. coli or the native pBD2 without the his-tag in pig, which meant that the polyclonal antibody had good specificity.

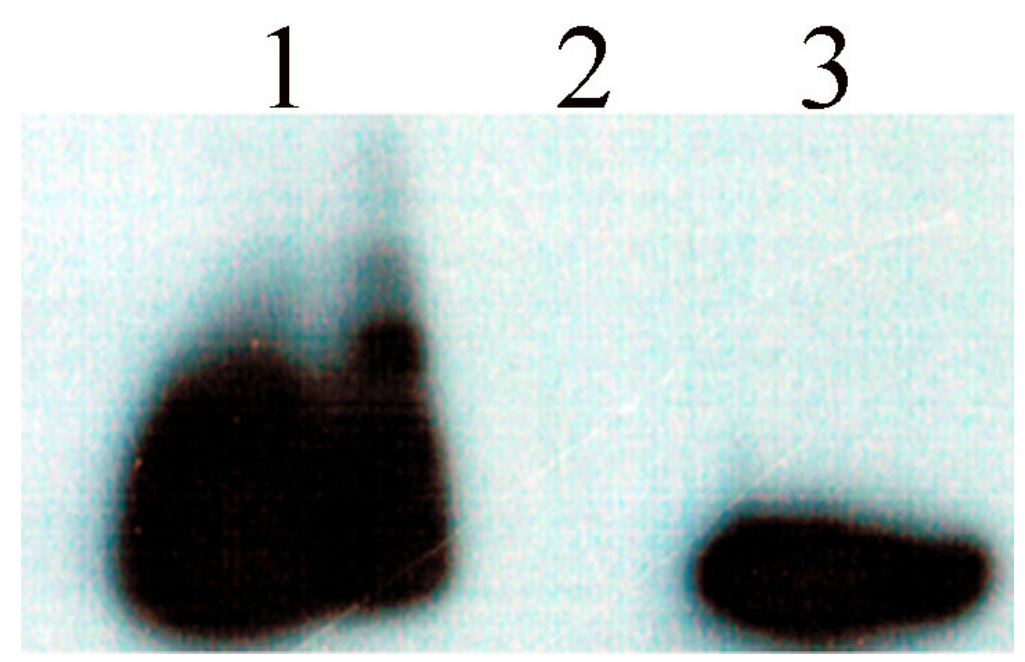

Figure 4. Specificity analysis of the polyclonal antibody by western blotting. Lane 1 shows total protein samples from $\mathrm{BL21}(\mathrm{DE} 3)-\mathrm{pET}-\mathrm{pBD2}$, lane 2 show total protein samples from E. coli BL21(DE3), and lane 3 shows proteins from porcine kidney tissue.

\section{Tissue immunohistochemistry assay}

Porcine tongue, liver, kidney, small intestine (including duodenum and jejunum), and large intestine (rectum) were used as samples to test the distribution of pBD2 in pig using the anti-pBD2 antibody. Immunohistochemistry analysis revealed the presence of pBD2 in stratified squamous epithelium in tongue (Figure 5b), interlobular arteries, and veins between hepatic lobules in the liver (Figure 5d), inner and outer epithelial cells in renal tubules in the kidney (Figure 5f), epithelial cells in the intestinal glands and glandular lumen in the small intestine (duodenum and jejunum), and large intestine (rectum) (Figure $5 \mathrm{~h}, \mathrm{j}$, and I). In contrast, no staining was observed in tissues in the absence of the primary polyclonal antibody (Figure $5 \mathrm{a}, \mathrm{c}, \mathrm{e}, \mathrm{g}$, i, and k). Thus, the polyclonal antibody is able to recognize endogenous pBD2 in tissues and may be used as a useful tool to study the function and mechanism of $\mathrm{pBD} 2$. This is the first time that $\mathrm{pBD} 2$ has been identified in porcine tissues at the protein level. 


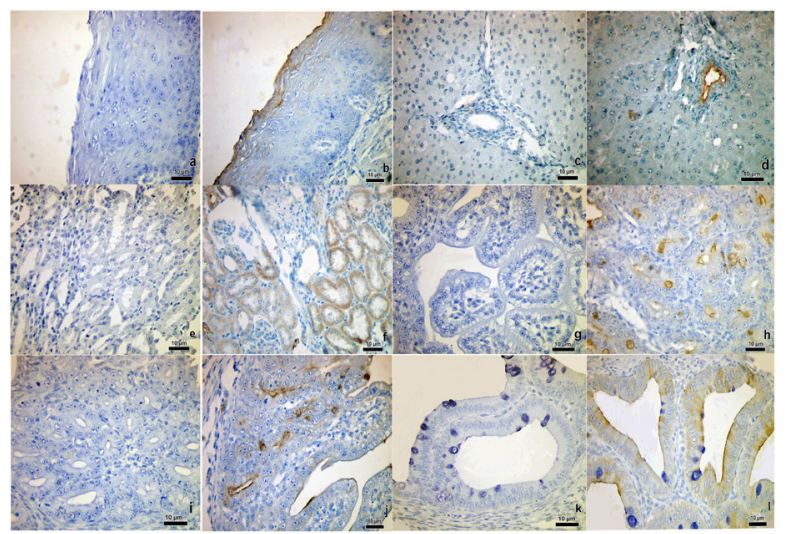

Figure 5. Immunohistochemical staining in some porcine tissues. Images a, c, e, g, i, and k show tongue, liver, kidney, duodenum, jejunum, and rectum tissue, respectively, which were incubated with serum from non-immunized rabbits as the primary antibody, followed by HRP-labeled goat anti-rabbit IgG. Images b, d, f, h, j. and I show tongue, liver, kidney, duodenum, jejunum, and rectum tissue, respectively, which were incubated with purified anti-pBD2 polyclonal antibody, followed by HRP-labeled goat anti-rabbit IgG. All tissue samples were stained with diaminobenzidine (DAB) and counterstained with hematoxylin. Brown staining indicates the presence of pBD2.

\section{DISCUSSION}

Infectious diseases are responsible for significant economic loss in the porcine industry, and multidrug-resistant bacteria are a serious global problem, which affect the morbidity and mortality of infected pigs. Therefore, it is important to find new antimicrobial treatments for use in these diseases. Studies showed pBD2 to have high antimicrobial activity against bacteria, including multidrug-resistant bacteria isolated from sick fowl (Veldhuizen et al., 2008; Li et al., 2013a), which makes it good candidate for antibiotic development. Furthermore, pBD2 showed low hemolytic activity, high salt-tolerance, and strong thermal stability, which are important properties for its practical application (Li et al., 2013a). However, the anti-bacterial mechanism of pBD2 and its role in the immune system are unclear. In addition, pBD2 antibodies are not commercially available. Thus, in this study, we prepared and characterized an anti-pBD2 polyclonal antibody.

Based on the BL21(DE3)-pET-pBD2 strain, which was constructed in our lab, his-pBD2 was expressed, purified, and used to immunize rabbits to prepare polyclonal antibody. The polyclonal antibody was precipitated with different concentrations of ammonium sulfate, and further purified by DEAE cellulose chromatography. The purified antibodies showed high sensitivity, with a titer as high as 204,800 by ELISA, and they specially recognized pBD2, whether it was the his-tagged fusion protein in E. coli or the native protein in pigs, as assessed by western blotting. Furthermore, the antibody specifically reacted with pBD2 in different pig tissues, and immunohistochemistry analyses revealed the distribution of pBD2 in the tongue, liver, kidney, small intestine (duodenum and jejunum), and large intestine (rectum) of Large White pigs. The results revealed a more detailed distribution of pBD2 at the protein level compared with that reported at the RNA level (Chen et al., 2010). Therefore, the prepared polyclonal antibody should be a useful tool for further studies.

In conclusion, in this study, purified his-pBD2 was used to prepare a polyclonal antibody. The anti-pBD2 antibody was purified, and it showed high sensitivity and specificity. The purified anti-pBD2 antibody reacted with the expressed fusion protein in $E$. coli and with the native protein in pigs. Furthermore, the distribution of pBD2 protein was identified in different pig tissues. The 
prepared polyclonal antibody should be a useful tool for the further study of pBD2, including its expression and distribution in various tissues, as well as its function and mechanism of action in the immune system.

\section{Conflicts of interest}

The authors declare no conflict of interest.

\section{ACKNOWLEDGMENTS}

(\#31101694).

Research supported by grant from the National Natural Science Foundation of China

\section{REFERENCES}

Auvynet $C$ and Rosenstein $Y$ (2009). Multifunctional host defense peptides: antimicrobial peptides, the small yet big players in innate and adaptive immunity. FEBS J. 276: 6497-6508.

Chen J, Qi S, Guo R, Yu B, et al. (2010). Different messenger RNA expression for the antimicrobial peptides beta-defensins between Meishan and crossbred pigs. Mol. Biol. Rep. 37: 1633-1639.

Choi MK, Le MT, Nguyen DT, Choi H, et al. (2012). Genome-level identification, gene expression, and comparative analysis of porcine b-defensin genes. BMC Genet. 13: 98.

Ganz T (2003). Defensins: antimicrobial peptides of innate immunity. Nat. Rev. Immunol. 3: 710-720.

Ganz T (2004). Defensins: antimicrobial peptides of vertebrates. C. R. Biol. 327: 539-549.

Gao CY, Xu TT, Zhao QJ and Li CL (2015). Codon optimization enhances the expression of porcine beta-defensin-2 in Escherichia coli. Genet. Mol. Res. 14: 4978-4988

Gao Y, Yang M, Peng C, Li X, et al. (2012). Preparation of highly specific anti-zearalenone antibodies by using the cationic protein conjugate and development of an indirect competitive enzyme-linked immunosorbent assay. Analyst 137: $229-236$.

Hancock RE (2001). Cationic peptides: effectors in innate immunity and novel antimicrobials. Lancet Infect. Dis. 1: $156-164$.

Klüver E, Adermann K and Schulz A (2006). Synthesis and structure-activity relationship of beta-defensins, multi-functional peptides of the immune system. J. Pept. Sci. 12: 243-257.

Kroll JJ, Eichmeyer MA, Schaeffer ML, McOrist S, et al. (2005). Lipopolysaccharide-based enzyme-linked immunosorbent assay for experimental use in detection of antibodies to Lawsonia intracellularis in pigs. Clin. Diagn. Lab. Immunol. 12: 693-699.

Lehrer RI and Ganz T (2002). Defensins of vertebrate animals. Curr. Opin. Immunol. 14: 96-102.

Li CL, Zhao YC, Song XY, Huang XX, et al. (2013a). Molecular Cloning, Expression and Characterization of the Porcine beta Defensin 2 in E. coli. Protein Pept. Lett. 20: 715-723.

Li J, Wu R, Chen H, Zhou Y, et al. (2013b). The cloning and characterization of the Enolase2 gene of Gekko japonicus and its polyclonal antibody preparation. Int. J. Mol. Sci. 14: 8787-8800.

Liu Q, Luo Y, Li X, Yuan L, et al. (2013). Preparation and characterizations of polyclonal antibodies against STAR protein QKI7b. Appl. Biochem. Biotechnol. 169: 2273-2280.

Liu X, Wu Y, Li M, Chen S, et al. (2009). Production of polyclonal antibody against interleukin-33 and assessment of its distribution in murine liver and lung. J. Biomed. Biotechnol. 2009: 729197.

Nozdrachev AD, Kolosova LI, Moiseeva AB and Ryabchikova OV (2006). The role of defensin NP-1 in restoring the functions of an injured nerve trunk. Neurosci. Behav. Physiol. 36: 313-315.

Parham P, Androlewicz MJ, Brodsky FM, Holmes NJ, et al. (1982). Monoclonal antibodies: purification, fragmentation and application to structural and functional studies of class I MHC antigens. J. Immunol. Methods. 53: 133-173.

Peschel A and Sahl HG (2006). The co-evolution of host cationic antimicrobial peptides and microbial resistance. Nat. Rev. Microbiol. 4: 529-536.

Sang Y, Patil AA, Zhang G, Ross CR, et al. (2006). Bioinformatic and expression analysis of novel porcine beta-defensins. Mamm. Genome. 17: 332-339.

Sang Y, Ruchala P, Lehrer RI, Ross CR, et al. (2009). Antimicrobial host defense peptides in an arteriviral infection: differential peptide expression and virus inactivation. Viral Immunol. 22: 235-242.

Selsted ME and Ouellette AJ (2005). Mammalian defensins in the antimicrobial immune response. Nat. Immunol. 6: 551-557. 
Shi J, Zhang G, Wu H, Ross C, et al. (1999). Porcine epithelial beta-defensin 1 is expressed in the dorsal tongue at antimicrobial concentrations. Infect. Immun. 67: 3121-3127

Veldhuizen EJ, Rijnders M, Claassen EA, van Dijk A, et al. (2008). Porcine $\beta$-defensin 2 displays broad antimicrobial activity against pathogenic intestinal bacteria. Mol. Immunol. 45: 386-394.

Wu C, Wang Y, Zou M, Shan Y, et al. (2007). Prokaryotic expression, purification, and production of polyclonal antibody against human polypeptide $\mathrm{N}$-acetylgalactosaminyltransferase 14. Protein Expr. Purif. 56: 1-7.

Xin T, Yang H, Wang N, Wang F, et al. (2013). Limitations of the BP26 protein-based indirect enzyme-linked immunosorbent assay for diagnosis of Brucellosis. Clin. Vaccine Immunol. 20: 1410-1417.

Xiong W, Luo Y, Zhang C, Tan D, et al. (2012). Expression, purification of recombinant human mitochondrial transcription termination factor 3 (hMTERF3) and preparation of polyclonal antibody against hMTERF3. Appl. Biochem. Biotechnol. 167: 2318-2329.

Yang D, Chertov O, Bykovskaia SN, Chen Q, et al. (1999). Beta-defensins: linking innate and adaptive immunity through dendritic and T cell CCR6. Science. 286: 525-528.

Yang D, Liu ZH, Tewary P, Chen Q, et al. (2007). Defensin participation in innate and adaptive immunity. Curr. Pharm. Des. 13: 3131-3139.

Zhang G, Ross CR and Blecha F (2000). Porcine antimicrobial peptides: new prospects for ancient molecules of host defense. Vet. Res. 31: 277-296. 